\title{
Hadronic Total Cross-sections Through Soft Gluon Summation in Impact Parameter Space
}

\author{
A. Grau ${ }^{1}$, G. Pancheri ${ }^{2}$, Y.N. Srivastava ${ }^{3}$ \\ 1) Departamento de Fúsica Teórica y del Cosmos, Universidad de Granada, Spain \\ 2) INFN, Laboratori Nazionali di Frascati, P.O. Box 13, I-00044 Frascati, Italy \\ 3) Physics Department and INFN, University of Perugia, Perugia, Italy
}

October 11, 2018

\begin{abstract}
The Bloch-Nordsieck model for the parton distribution of hadrons in impact parameter space, constructed using soft gluon summation, is investigated in detail. Its dependence upon the infrared structure of the strong coupling constant $\alpha_{s}$ is discussed, both for finite as well as singular, but integrable, $\alpha_{s}$. The formalism is applied to the prediction of total proton-proton and proton-antiproton cross-sections, where screening, due to soft gluon emission from the initial valence quarks, becomes evident.
\end{abstract}

\section{Introduction}

In this paper we address some phenomenological implications of the infrared behaviour of the strong coupling constant $\alpha_{s}[$ [1]. In particular, we examine some models for the total proton-proton and proton-antiproton cross-sections and show the dependence of the rise with energy of the cross-section upon the small $k_{t}$ behaviour of $\alpha_{s}$, through the mechanism of soft gluon summation. In a previous paper [2], soft gluon summation techniques have been applied to develop a model for the impact parameter distribution of partons in hadronic collisions. According to this model, the distribution in impact parameter 
space (b-distribution) is the Fourier transform of the transverse momentum distribution of the colliding parton pair, and is obtained by using the BlochNorsdieck technique for soft gluon summation, developed some time ago to describe hadronic transverse momentum distributions [3, 4, 5, 6]. This model for the $b$-distribution of partons is used in the context of eikonal models for total cross-sections, and in particular in the context of the eikonal mini-jet models, where the rise with energy is driven by the jet cross-section calculated from QCD. In order to make full use of QCD for this particular problem, it is necessary that not only the energy dependence be derived from QCD, but also the $b$-dependence, at least for what concerns the hard part of the cross-section : it may otherwise be possible to obscure the difficulties of QCD inspired models through various parameters which are still present in it. One of the difficulties is that the QCD cross-section rises too fast with energy to be able to accomodate both the early rise (around $\sqrt{s}=10 \div 20 \mathrm{GeV}$ ) and the high energy behaviour at $\sqrt{s} \geq 200 \div 300 \mathrm{GeV}$ and beyond. In some mini-jet models the too abrupt rise of the mini-jet cross-section is softened by modifying the small $\mathrm{x}$-behaviour of the parton densities. Our alternative proposal, discussed in detail in this paper, is to regulate the rise of the crosssection through soft gluon emission.

In Sect. 2 we present a brief description of the eikonal mini-jet model. In Sects. $3 \div 6$ we shall analyze the structure of the Block-Nordsieck model for the $b$-distribution of partons, first recalling the main features of the model, and then studying, analytically as well as numerically, its behaviour employing various phenomenological models for the $k_{t} \rightarrow 0$ behaviour of the strong coupling constant $\alpha_{s}$. In all cases, we shall compare our results with those from a model in which the matter distribution of partons is obtained from the electromagnetic form factor of the colliding hadrons. In the last two sections, Sects. 7 and 8, we shall study the predictions of the Bloch-Nordsieck model for total cross-sections and shall compare our results for proton-proton and proton-antiproton collisions with other models and present data. It will be shown that the model, with a singular but otherwise integrable behaviour of $\alpha_{s}$, is flexible enough to accomodate both the early rise with energy as well as present data from the Tevatron. 


\section{Eikonal mini-jet model for total cross-sections}

Ever since the first observation of the rise of proton-proton total cross-section, the suggestion was advanced that such rise was due to the increasing (with energy) number of hard collisions taking place among the hadron constituents [0]. This ansätz was subsequently quantified by the mini-jet model, which proposes to calculate the total inelastic cross-section from the jet cross-section obtained from QCD [8, 9]. The unitarized version of the mini-jet model is represented by the eikonalized minijet model[10, 11, 12], in which the total cross-section is given by

$$
\sigma_{t o t}=2 \int d^{2} \vec{b}\left[1-e^{-n(b, s) / 2}\right]
$$

with

$$
n(b, s)=A(b)\left[\sigma_{s o f t}+\sigma_{j e t}\right]
$$

and $A(b)$ a function which represents the impact parameter distribution of partons in the collision. In its most intuitive formulation, the overlap is obtained from the Fourier transform of the electromagnetic form factors $\mathcal{F}_{1}$ and $\mathcal{F}_{2}$ of the colliding hadrons, i.e.

$$
A_{F F}(b)=\frac{1}{(2 \pi)^{2}} \int d^{2} \vec{q} e^{i b \cdot q} \mathcal{F}_{1}(q) \mathcal{F}_{2}(q)
$$

The model which uses this overlap function, hereafter called the form factor (FF) model, although attractive, is of course not parameter free, as it depends on the scale parameters characterizing the form factors.

The two cross-section $\sigma_{\text {soft }}$ and $\sigma_{\text {jet }}$ are respectively a non-perturbative term and a function of energy obtained by integrating the QCD jet crosssection from a minimum $p_{t}$ value, $p_{t m i n}$, to the maximum kinematically allowed. This quantity increases with energy at fixed $p_{t m i n}$, depending upon various QCD controlled quantities like the parton densities, in particular, and very strongly, upon the small x-behaviour of the gluon densities. In fact, the kinematic lower limit in the $\mathrm{x}$-integration for the jet cross-section is given by $x_{\text {min }}=4 p_{\text {tmin }}^{2} / s$, and it can be as low as $10^{-6}$ at Tevatron energies. With such small $\mathrm{x}$-values, the jet cross-section grows much too rapidly as

$s$ increases and so does the eikonalized cross-section. In order to apply the mini-jet model to data, a screening effect is obtained either using the much 
less dangerous limit $\sqrt{x_{\min }}$ or softening the small-x singularity with a cutoff parameter. In this way, the above model can reproduce the energy rise, but with some further modifications, notably in $A(b)$. In particular, in order to obtain reasonable agreement with the data it is also necessary to modify the simple form factor model, by allowing for different values of the scale parameters for the low and high energy region.

Our approach is different. We believe that the function $A(b)$ is not a constant in energy and for the hard part of the collisions we have proposed a model in which soft gluon emission is responsible for the b-distribution of the colliding partons. Since the overall soft gluon emission summation is energy dependent, we expect such model can modify and complement the mini-jet model description of total cross-sections.

\section{Bloch-Nordsieck formalism in impact pa- rameter space}

The Bloch-Nordsieck distribution depends upon the energies of the colliding quarks and gluons and is thus, although mildly, energy dependent. In this

section we shall recapitulate the main features of this model, whose general structure was derived in ref. [2]. As described, our proposed impact parameter space distribution for a pair of partons $\mathrm{i}$ and $\mathrm{j}$ is given by

$$
A_{B N}=\frac{e^{-h(b ; M, \Lambda)}}{2 \pi \int b d b e^{-h(b ; M, \Lambda)}}
$$

where

$$
h(b ; M, \Lambda)=\frac{2 c_{i j}}{\pi} \int_{0}^{M} \frac{d k_{t}}{k_{t}} \alpha_{s}\left(k_{t}^{2}\right) \ln \frac{M+\sqrt{M^{2}-k_{t}^{2}}}{M-\sqrt{M^{2}-k_{t}^{2}}}\left[1-J_{0}\left(k_{t} b\right)\right]
$$

with $c_{i j}=4 / 3$ for a quark-antiquark pair. In eqs.(4,5) the hadronic scale M accounts for the maximum energy allowed to each single soft gluon emitted in the collision. This quantity depends upon the energy of the colliding parton pair and, through this, upon the energy of the initial colliding hadrons. The main point of our model is that soft gluon emission destroys the collinearity of the colliding partons. Let us distinguish now between valence partons and 
gluons or sea quarks. In first approximation, gluons and sea quarks can be considered as having the same non-collinearity as the initial valence quarks which emit them during the hadronic collision (a different case will be that of the photons, which we shall discuss in a different paper). To leading order we can now assume that the impact parameter distribution of all type of parton pairs is the same as that of the valence quarks. This approximation is in the same spirit as the one for which the impact parameter distribution in the form factor model is given by the Fourier transform of the electromagnetic form factors, i.e. matter distribution follows charge distribution.

In the calculation of total cross-sections with the eikonalized mini-jet model, the distribution (4) appears convoluted with parton densities and jet cross-sections. In ref. [2], we proposed to write the average number of collisions at impact parameter $b$ as

$n(b, s)=n_{\text {soft }}(b, s)+\sum_{i, j,} \int \frac{d x_{1}}{x_{1}} \int \frac{d x_{2}}{x_{2}} f_{i}\left(x_{1}\right) f_{j}\left(x_{2}\right) \int d z \int d p_{t}^{2} A_{B N}\left(b, q_{\max }\right) \frac{d \sigma}{d p_{t}^{2} d z}$

where $f_{i}$ are the quark densities in the colliding hadrons, $q_{\max }$ is the maximum transverse momentum allowed by kinematics to a single gluon emitted by the initial $q \bar{q}$ pair, $z=\hat{s}_{j e t} /\left(s x_{1} x_{2}\right)$, and $\frac{d \sigma}{d p_{t}^{2} d z}$ is the differential cross-section for process

$$
q \bar{q} \rightarrow \text { jet jet }+X
$$

for a given $p_{t}$ of the produced jets with c.m. energy $\sqrt{\hat{s}_{j e t}}$. The jet pair in process (7) is the one produced through any subprocess initiated by the valence quark-antipark pair, thus it could be gluon jets, or quark jets. In Fig. 1 we show some typical subprocesses which contribute to (7). For high energy and low $p_{t}$, most of the jets are produced through scattering of gluons emitted by a valence quark pair which continues undetected after emission. In principle, an exact calculation of this model for $n(b, s)$ would require to know $A\left(b, q_{\max }\right)$ for each subenergy $\hat{s}$ of the quark-antiquark pair because for process (7)

$$
q_{\max }(\hat{s})=\frac{\sqrt{\hat{s}}}{2}\left(1-\frac{\hat{s}_{j e t}}{\hat{s}}\right)
$$

and then one would need to calculate $n(b, s)$ for each s value, through convolution for all parton densities and all subprocesses. This procedure is at present unpractical for this problem, since the $b$-parameter dependence applies to the 
valence quark

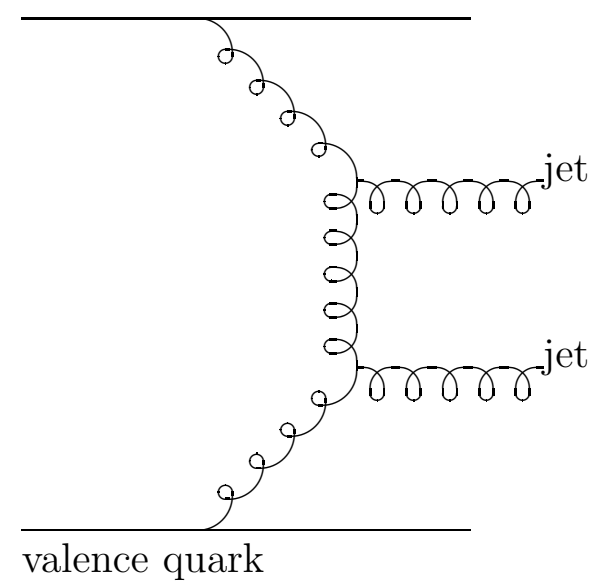

valence quark

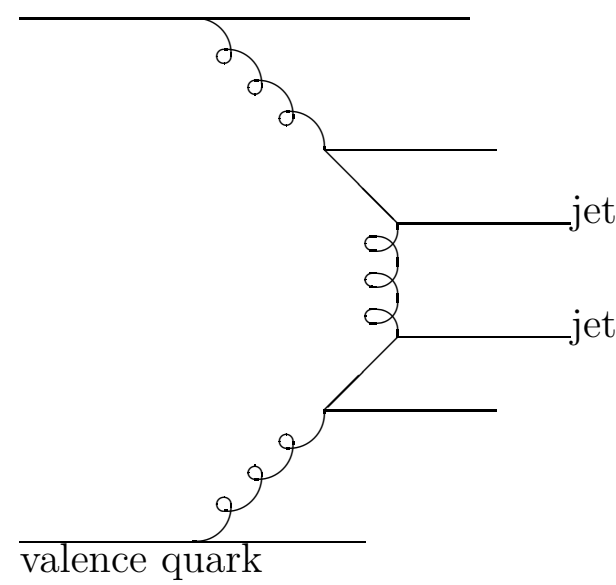

Figure 1: Two typical subprocesses contributing to the rise of the total proton-proton cross-section

initial valence pair. What is available, through various parametrizations, is parton densities after $Q^{2}$ evolution, for all type of partons, whereas the above formulation would require to apply corrections and evolution in expressions which depend upon the impact variable $b$. In any case, before recommending to embarque in such a time-consuming integration, one can study the properties of the proposed model, adopting some approximations, which allow for phenomenological calculations. The approximation described in [2] is

$$
n(b, s)=n_{\text {soft }}+A_{B N} \sigma_{j e t}\left(s, p_{\text {tmin }}\right)
$$

where $A_{B N}$ is the function $A_{B N}\left(b,<q_{\max }>\right)$ evaluated at the value $M=<$ $q_{\max }>$, obtained by averaging over all parton densities and jet subprocesses. In the next section we shall evaluate $\left\langle q_{\max }>\right.$ for different energies of the colliding hadrons and for different $p_{t m i n}$ values. 


\section{The scale dependence : $q_{\max }$}

Using the expression

$$
M \equiv<q_{\max }(s)>=\frac{\sqrt{s}}{2} \frac{\sum_{i, j} \int \frac{d x_{1}}{x_{1}} f_{i / a}\left(x_{1}\right) \int \frac{d x_{2}}{x_{2}} f_{j / b}\left(x_{2}\right) \sqrt{x_{1} x_{2}} \int d z(1-z)}{\sum_{i, j} \int \frac{d x_{1}}{x_{1}} f_{i / a}\left(x_{1}\right) \int \frac{d x_{2}}{x_{2}} f_{j / b}\left(x_{2}\right) \int(d z)}
$$

with $z_{\min }=4 p_{\text {tmin }}^{2} /\left(s x_{1} x_{2}\right)$, one can plot the quantity $M$ as a function of $\sqrt{s}$ for different values of $p_{\text {tmin }}$. This is shown in Fig.2, where we have used $\mathrm{GRV}(\mathrm{LO})$ 13 parton densities for proton proton collisions.

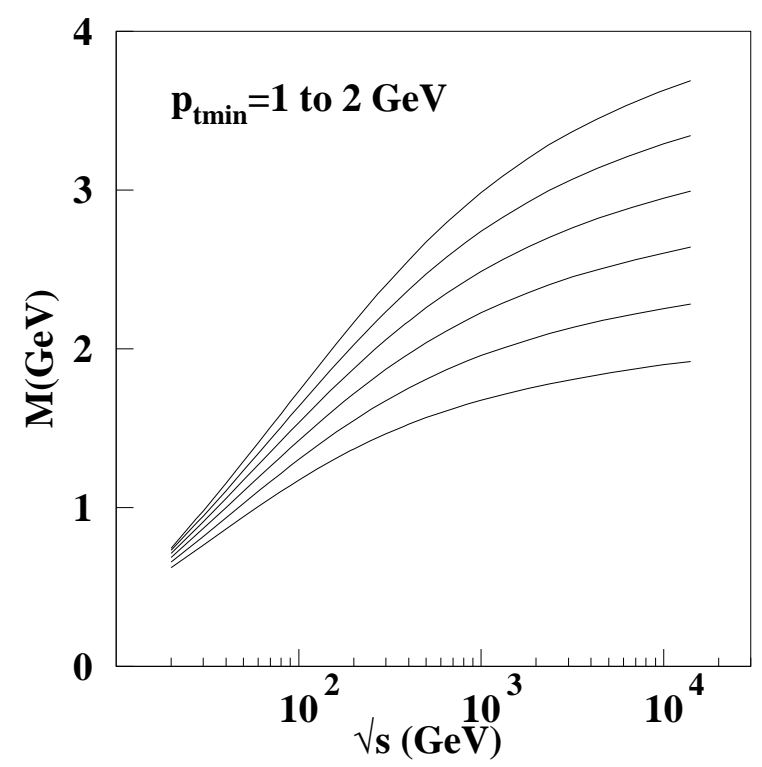

Figure 2: The maximum value kinematically allowed for the transverse momentum of the single gluon, averaged over densities (GRV-LO parametrization) and for different $p_{\text {tmin }}$ values, as a function of the c.m. energy of the colliding protons.

One sees that, for $\sqrt{s} \approx 50 \div 10^{4} \mathrm{GeV}$, the range of values for $M$ is between 0.5 and $4 \mathrm{GeV}$ for $p_{\text {tmin }}=1 \div 2 \mathrm{GeV}$. For these typical values, one can now calculate $h(b ; M, \Lambda)$ and subsequently $A(b, M)$. Our point of interest in this paper is also to relate the rate of rise of the total cross-section with the behaviour of $\alpha_{s}$ in the infrared region. The stronger the singularity as $k_{t} \rightarrow 0$, 
the larger $h(b ; M, \Lambda)$, the faster $A_{B N}$ goes to zero and the stronger will be the suppression produced by soft gluon emission. We shall now quantify this statement with numerical calculations.

\section{$5 \quad \alpha_{s}$ dependence in the function $h(b ; M, \Lambda)$}

We start by showing how the b-dependence of $h(b ; M, \Lambda)$ varies according to the behaviour of $\alpha_{s}$ in the very low $k_{t}$ region. Because of the many uncertainties we shall work with the one-loop expression for $\alpha_{s}$ and shall use two different models, each of them characterized by a set of parameters, i.e. the frozen $\alpha_{s}$ model used in [14, 15] where

$$
\alpha_{s}\left(k_{t}^{2}\right)=\frac{12 \pi}{33-2 N_{f}} \frac{1}{\left.\ln \left[\left(k_{t}^{2}+a^{2} \Lambda^{2}\right) / \Lambda^{2}\right)\right]}
$$

which depends upon the parameter set $\{\Lambda, a\}$ and in which $\alpha_{s}$ goes to a constant value as $k_{t}$ goes to zero. An altogether different model is the singular $\alpha_{s}$ model, described in [2] with

$$
\alpha_{s}\left(k_{t}^{2}\right)=\frac{12 \pi}{\left(33-2 N_{f}\right)} \frac{p}{\ln \left[1+p\left(\frac{k_{t}^{2}}{\Lambda^{2}}\right)^{p}\right]}
$$

which coincides with the usual one-loop expression for large values of $k_{\perp}$, while going to a singular limit for small $k_{\perp}$. In this model, $\alpha_{s}$ depends upon the parameter set $\{\Lambda, p\}$. The singular expression of eq.(12) is inspired by the Richardson potential [16] used in quarkonium spectroscopy. The Richardson potential can be connected to a singular $1 / k_{\perp}^{2}$ behaviour of $\alpha_{s}$ in the infrared limit, a singularity which is not dangerous in bound state problems, where the Schroedinger equation selects only those solutions for which the momentum is fixed by the stability condition. For this problem, and as discussed in [2], the expression we have chosen should be considered as a toy model, in which the singular behaviour of $\alpha_{s}$ (if any) can be modulated through the singularity parameter $p$. One should also notice that the singular limit of the above equation is not an observable. Phenomenologically, one never measures $\alpha_{s}$ in the $k_{\perp} \rightarrow 0$ limit, since this limit corresponds to emission

of a very soft gluon, in which case summation, and hence integration over $k_{\perp}$, is mandatory. In other words, what really matters is the integrability 
of the function, since observable quantitites (soft gluons are observed only as overall energy momentum imbalance carried away by soft particles, but not measured individually) always involve an integration over the infrared region. In what follows we shall always use the set $a=2, \Lambda=0.2 \mathrm{GeV}$ for the frozen $\alpha_{s}$ model, whereas for the singular case, while we shall vary the singularity parameter $p$, we shall adopt the value $\Lambda=0.1 \mathrm{GeV} 17$.

Let us now examine the function $h(b ; M, \Lambda)$. This function does not allow for a closed form expression, and needs to be numerically evaluated. Useful analytical approximations can be found in the appendix.

The dependence of the function $h(b ; M, \Lambda)$ upon the infrared behaviour of $\alpha_{s}$ is shown in Fig. 3, where we have plotted in the same graph the exactly integrated expression for the function $h(b, M, \Lambda)$ for the frozen case, eq.(11), and for the singular case, eq.(12), for two values of the parameter p. For each

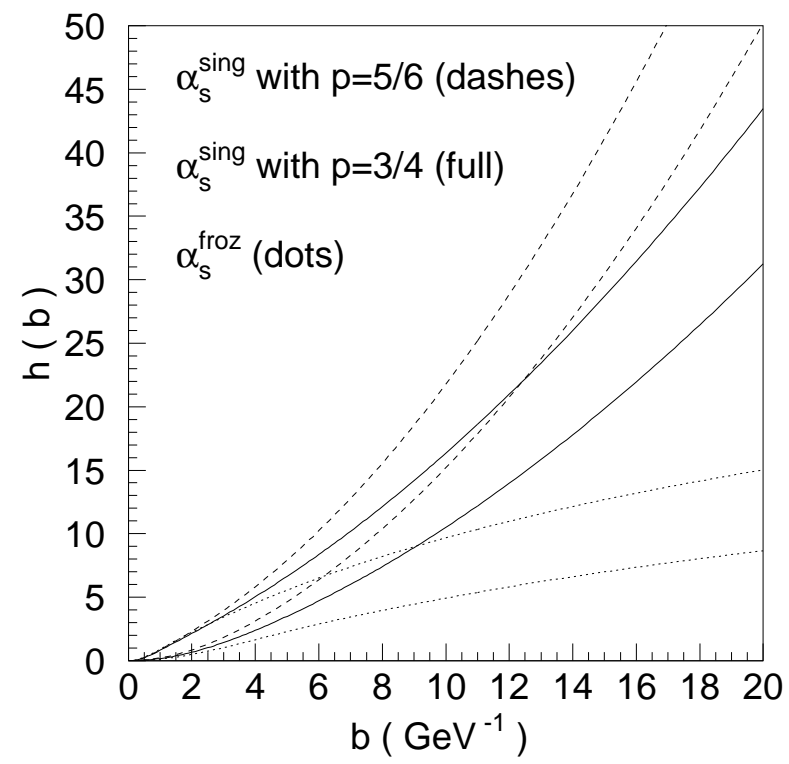

Figure 3: Comparison between numerically integrated expressions for $h(b, M, \Lambda)$ for singular and frozen $\alpha_{s} . M=1$ (lower) and $4 \mathrm{GeV}$ (upper).

case, we have evaluated $h(b)$ for the two values $M=1$ and $4 \mathrm{GeV}$, which correspond to the interesting range $\sqrt{s}=50 \div 10^{4} \mathrm{GeV}$, for $p_{\text {tmin }}=1 \div 2 \mathrm{GeV}$ 
(see Fig. 2). Although at very small $b\left(b \leq 0.2 \mathrm{GeV}^{-1}\right)$ the values are not very different, at larger $b$-values there is an increasing discrepancy between the two formulations. The large $b$ region below $\approx 10 \mathrm{GeV}^{-1}$ is the one which matters most for the total cross-section analysis, where the figures then show that the infrared behaviour of $\alpha_{s}$ plays an important role in the rise of the cross-section. In the next sections we shall study the difference in $A(b)$ and then in the number of collisions, given the same jet-cross-section.

\section{The overlap function $A(b)$}

In this section, we shall calculate numerically $e^{-h(b ; M, \Lambda)}$ and the normalized $A(b)$, for the two cases, frozen and singular $\alpha_{s}$. We show in Figs.4, 5 the normalized function $A(b)$ for the frozen and singular $\alpha_{s}$ possibilities, using, for the latter case, three different values of the parameter $p$ which regulates the singularity. In both figures we also show the comparison with the

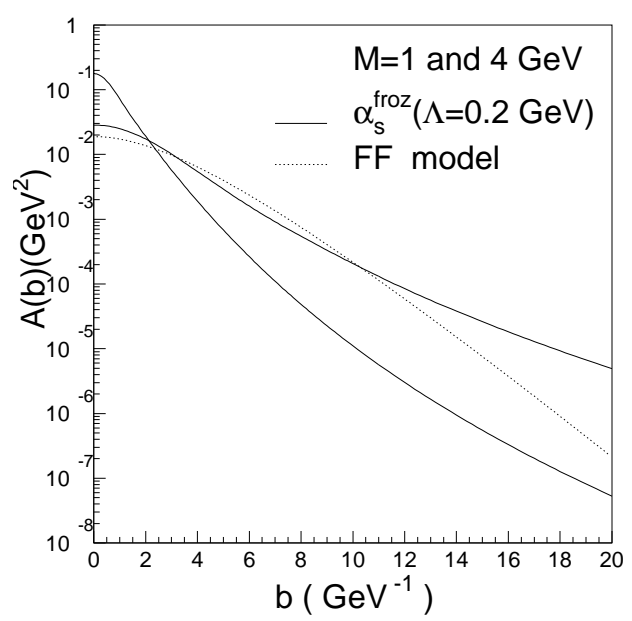

Figure 4: The overlap function $A_{B N}(b)$ for frozen $\alpha_{s}$ and comparison with the Form Factor model

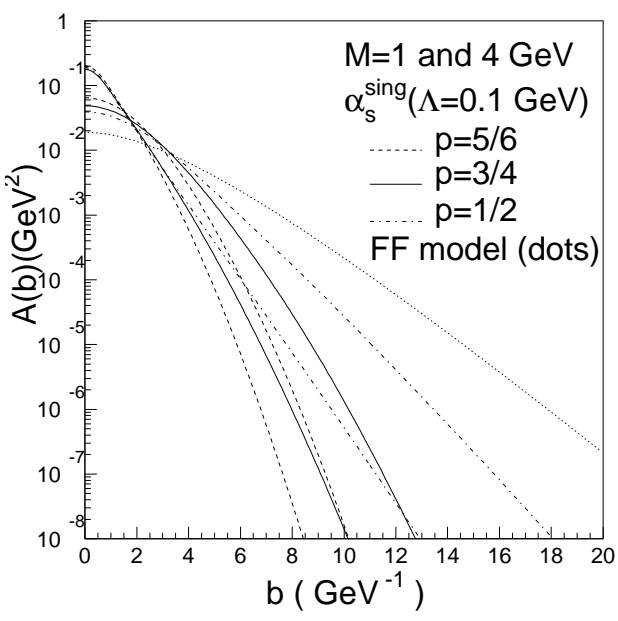

Figure 5: As in Fig. 14 for singular $\alpha_{s}$, for various values of the parameter $p$.

function $A(b)$ in the form factor model, according to which matter density in the proton is given by the electromagnetic form factor. With the usual 
parametrization

$$
\mathcal{F}_{\text {proton }}(q)=\left(\frac{\nu^{2}}{q^{2}+\nu^{2}}\right)^{2} \quad \nu^{2}=0.71 G e V^{2}
$$

the overlap function $A(b)$ in the form factor model has the expression

$$
A_{F F}(b)=\frac{\nu^{2}}{96 \pi}(\nu b)^{3} K_{3}(\nu b)
$$

In each figure, the various curves correspond to varying the scale $M$ as described in the previous section, so that they include a range of energies $\sqrt{s}=50 \div 10^{4} \mathrm{GeV}$ for a range of $p_{\text {tmin }}$ between 1 and $2 \mathrm{GeV}$. We see that the frozen $\alpha_{s}$ case is more similar to the form factor model, especially at low medium energies, $(50 \rightarrow 100 \mathrm{GeV})$, when the proton is not yet exhibiting the full QCD behaviour. This is different from the singular case, where the function $A(b)$ is always falling with energy more than in the form factor model. The more singular $\alpha_{s}$ as $k_{t} \rightarrow 0$ (larger $p$ values), the more concentrated at small impact parameter is the overlap function and hence the less important the large b-values. This will have as physical consequence that as the c.m. energy increases, the non-collinearity of the initial state due to soft gluon emissions will accordingly increase. Clearly this will signify a much more noticeable effect of soft gluon straggling on the total cross-section. We shall now see this effect on the average number of collisions $n(b, s)$.

\section{Average number of collisions}

In the eikonalized mini-jet model, the quantity which contains the energy dependence of the total cross-section, is the average number of collisions $n(b, s)$. At low c.m. energy of the colliding particles, this number is dominated by contribution from soft, non-perturbative type events, while the QCD component, mini-jet like, slowly rises, reaching a comparable size in the $200 \div 300 \mathrm{GeV}$ region. As mentioned in the first section, one can approximate the average number of collisions in the entire region as

$$
n(b, s)=n_{\text {soft }}(b, s)+n_{\text {hard }}(b, s)
$$

with

$$
n_{\text {soft }}(b, s)=A_{F F}(b) \sigma_{\text {soft }}(s)
$$


and

$$
n_{\text {hard }}(b, s)=A_{B N}(b ; M, \Lambda) \sigma_{j e t}\left(s, p_{\text {tmin }}\right)
$$

To study the b-behaviour, we shall introduce the soft term, by using the form factor model for $A(b)$ described in the previous section, which is consistent with a low energy model of the proton in which only valence quarks play a role in the scattering. In this model all the energy dependence comes from the cross-section term : we will parametrize $\sigma_{\text {soft }}$ so as to reproduce, through the eikonal, the low energy behaviour of the total proton-proton and proton anti-proton cross-sections. We found, as best fit to the low energy data with an eikonal formulation with $n_{\text {hard }}=0$

$$
\sigma_{\text {soft }}^{p p}=47+\frac{46}{E^{1.39}}
$$

and

$$
\sigma_{\text {soft }}^{p \bar{p}}=47+\frac{129}{E^{0.661}}+\frac{357}{E^{2.7}}
$$

where $E$ is the proton energy in the Laboratory system in $\mathrm{GeV}$ and the cross-sections are in $m b$. For $\sigma_{\text {jet }}$ we use GRV(LO) densities to evaluate the proton-proton jet cross-section and two different values of $p_{\text {tmin }}=1.2$ and $2 \mathrm{GeV}$, the latter being the one for which the total cross-section in the $\mathrm{FF}$ model passes through the CERN data points at $\sqrt{s}=546 \mathrm{GeV}$. In a subsequent section, when we shall try to fit the total cross-section data, we shall use other $p_{t m i n}$ values. We can now plot the entire $n(b, s)$ as a function of $\mathrm{b}$, for various values of the center of mass energy $\sqrt{s}$, which corresponds to various values of the scale $M$, as described in the first section. We show this behaviour for the frozen and singular $\alpha_{s}$ case in Figs.6, for $p_{t m i n}=2 \mathrm{GeV}$.

For the frozen $\alpha_{s}$ model, shown in Fig.6, the results are compared to a straightforward application of the form factor model, i.e. with

$$
n_{F F}(b, s)=A_{F F}(b)\left[\sigma_{s o f t}+\sigma_{j e t}\right]
$$

We see that at $\sqrt{s}=100 \mathrm{GeV}$, there is still no difference betwen the two models. On the other hand, as the energy increases, the BN model shows a stronger suppression of the large $b$-contribution. For the singular case, in order to show variations with the singularity parameter $p$, we plot in Fig.7 the result for different $p$-values. Notice that the $p$-dependence is related to the values of $k_{t}$ probed, i.e. by the $M$ values, which are smaller the smaller 


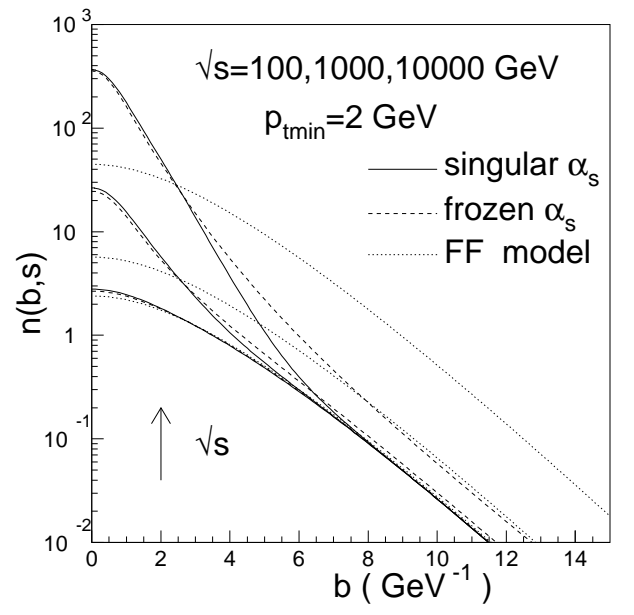

Figure 6: The average number of collisions for the frozen and singular $\alpha_{s}$ case in comparison with the FF model at various c.m. energy values.

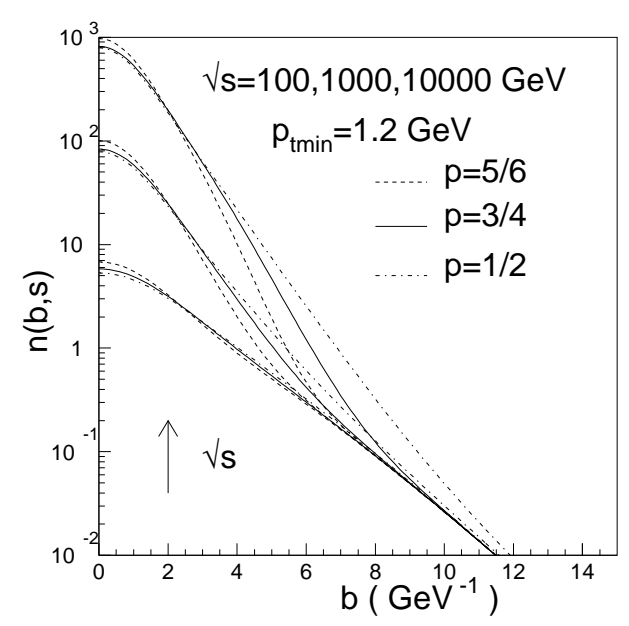

Figure 7: The average number of collisions for the singular $\alpha_{s}$ case for different values of the singularity parameter $p$.

$p_{\text {tmin }}$ is. Thus, for $p_{\text {tmin }}=2 \mathrm{GeV}$ for instance, there is very little difference among the various curves, at any given energy. This reflects the fact that the upper integration limit in eq.(5) is a relatively large $M(3 \div 4 \mathrm{GeV})$ value, so that the overall function is not very sensitive to the infrared region. It should be noted that for smaller $p_{\text {tmin }}$ values, like the ones actually used for fitting the total cross-sections in the next section, the dependence upon $p$ is much more noticeable. We show one such case in Fig.77.

The next figure, Fig.8, shows a break down of the average number of collisions into the soft and the hard component. In the present analysis we are not changing the soft component, which appears as the dash-dotted curve, and the figure shows, at a given high (LHC) energy, how the hard part would be different in the three models, i.e. in general more peaked at small $\mathrm{b}$ for the Bloch-Nordsieck model, and in particular falling faster the stronger the singularity of $\alpha_{s}$. At lower energies, where the mini-jet contribution is less important, these discrepancies would be much reduced. So, in this picture, while keeping a similar b-distribution at low energy, we quantitatively enhance small b-collisions at high energy, though QCD soft gluon emission. The change in the $b$-distribution introduced in the hard 


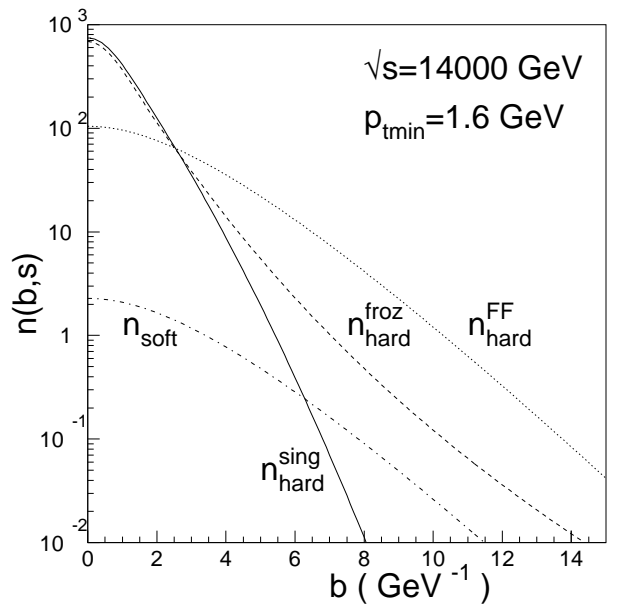

Figure 8: Soft and hard component of $n(b, s)$ in the three models described in the text.

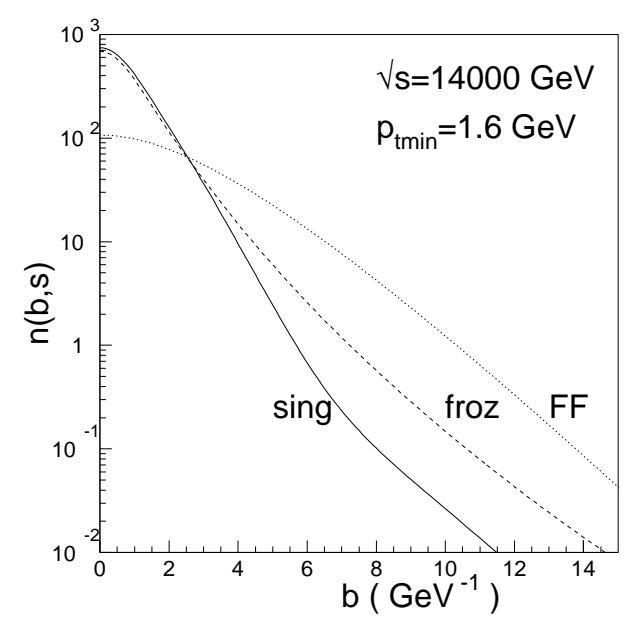

Figure 9: The average number of collisions in the form factor model and the Bloch Nordsieck model, at LHC energy.

component by the different models for $A(b)$ is responsible for the changed shape of $n(b, s)$ between the form factor and the other two models. The direct comparison among the three models is shown in Fig.(9) where the average number of collisions at $\sqrt{s}=14 \mathrm{TeV}$ is plotted for a choice of the various parameters as indicated. Apart from the change in shape, it can be noticed that the frozen $\alpha_{s}$ case corresponds to a behaviour intermediate between the form factor model, and the singular $\alpha_{s}$ case. We also see from this figure that the range of values of the $b$-parameter most important to the total cross-section calculation changes in the different models.

\section{Total cross-sections}

Before attempting the last relevant phenomenological exercise for the calculation of the total proton-proton and proton-antiproton cross-section, we shall first show how the integrand in eq.(11) changes with energy and which values of $b$ are most relevant for the calculation of the total cross-section in the various models for $A(b)$ we have just described. We must stress that this is not an optimization of the many parameters from which this model 
depends : rather an exercise to show how the Bloch-Nordsieck model for the impact parameter distribution affects the total cross-section behaviour in the eikonalized minijet model and how the behaviour of $\alpha_{s}$ in the infrared region is related to the rise of the total cross-section. This is done in Fig.10. The figure shows how much the integrand of eq.(贯) is peaked at different

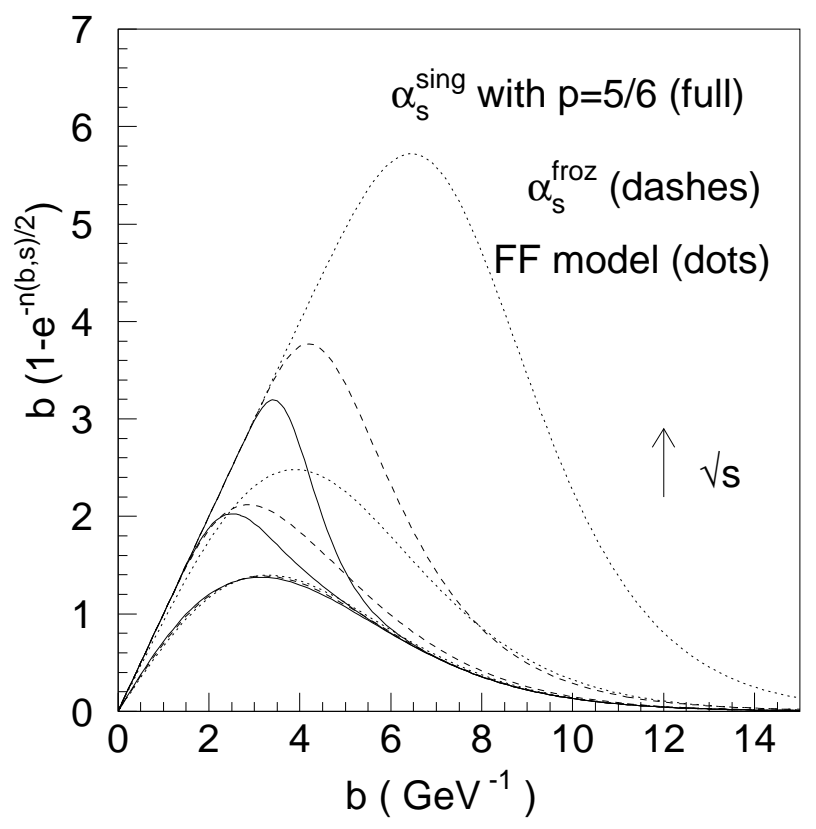

Figure 10: The integrand of the eikonal formulation for $\sigma_{t o t}$, for $p_{\text {tmin }}=$ $2 \mathrm{GeV}$ in the three different models described in the text, for a range of c.m. energy values $100,1000,10000 \mathrm{GeV}$

$b$-values as the energy increases, but also as the model for $A(b)$ changes. And it indicates that the rise with energy of the area under the curve, i.e. the cross-section, at the same energy shrinks for the more singular $\alpha_{s}$ behaviour.

Finally in Fig.11 we show the comparison of this model with proton- 
proton and proton-antiproton data. For proton-proton, we only show data up to ISR energies, since the existing data points in the $\mathrm{TeV}$ range are extrapolations from cosmic ray data [18] from $p$-air collisions and are partly model dependent [19]. For the proton-antiproton data, we have plotted all the data points published so far from the CERN $S p \bar{p} S$ 20] and FNAL 21 experiments. This introduces a larger band of uncertainty that it is usually shown, but the purpose of this paper is to indicate the potentiality of the Bloch-Nordsieck model rather than to do a best parameter fit, and we have opted for a comparison of our results with the full experimental picture.

We have studied three different formulations of the eikonal mini-jet model, one for the form factor model and two for the soft gluon summation model. To choose the parameters of the mini-jet description, we have selected those $p_{\text {tmin }}$ values which would ensure that the curve can reach the high energy points : for the form factor model this can be accomplished with $p_{\text {tmin }}=$ $2 \mathrm{GeV}$ but, as often stressed, with such value it is not possible to fit the early rise of the data. A lower value of $p_{t m i n}$ would on the other hand give curves which rise too much at higher energy and miss the points. Going to the soft gluon summation model, it must be noticed that since this model has an energy dependence in the b-behaviour in addition to the one in the jet-cross-section (common to all the models), one can expect that a smaller $p_{\text {tmin }}$ could be used, thus allowing for the earlier rise. In fact, the high energy data, for the frozen $\alpha_{s}$ model, can be met with $p_{t m i n}=1.6 \mathrm{GeV}$. Although with this value, the cross-section starts rising sooner than in the form factor model, still it is impossible to fit both the early rise as well as the high energy points. This model depends not only upon $p_{\text {tmin }}$ value, but also on the scale $a$ which regulates the infrared behaviour of $\alpha_{s}$ : the smaller $a$, the more singular the behaviour and the easier to fit the early rise. Finally, we show the results for the singular $\alpha_{s}$ case, with a particular choice of the parameter $p$ which regulates the singularity of $\alpha_{s}$. We can choose now a rather small value of $p_{\text {tmin }}$ to reproduce the early rise, since at higher energy the increased soft gluon emission reduces drastically the large$\mathrm{b}$ contribution to the cross-section and does not let it rise as much as in the other models. Our results are compared with a multiparameter fit from a QCD inspired model[12], which has recently been used to successfully predict photon photon total cross-sections 22]. These results are not very different from the ones obtained using the Regge-Pomeron exchange picture 23], but the model in [22] is closer in spirit to the one discussed here, with the energy 
rise due to the rise of the QCD jet cross-section.

The results of this figure shows that it is possible to have a rise in agreement both with the intermediate energy data as well as with the Tevatron data : this result is obtained using a single eikonal function, usual QCD parton densities and minijet cross-sections with $p_{\text {tmin }}$ in the $1 \div 2 \mathrm{GeV}$ range. To follow the beginning of the rise, one needs a rather low $p_{\text {tmin }}$. In general such low values imply too fast a growth of the total cross-section, in our case this fast growth is tampered by the increasing number of soft gluon emission phenomena at small $k_{t}$.

\section{Conclusions}

We have presented a detailed numerical analysis of a Bloch-Nordsieck approach to the impact parameter distribution of partons in the context of the eikonal mini-jet model for total hadronic cross-sections. We have shown that the proposed soft gluon summation expression plays an important role in softening the rise of the cross-section due to mini-jets and have studied the role which the infrared behaviour of $\alpha_{s}$ plays in it.

\section{Acknowledgments}

This work was supported in part by EEC-TMR Contract N.CT9800169 and by CICYT Contract N. AEN 96-1672.

\section{A Approximate Expressions for $h(b, M, \Lambda)$}

In this section, we show some analytic approximations to the function $h(b, M, \Lambda)$. Here we shall restrict our attention to values of $\mathrm{M}$ relevant to the total crosssection calculations, i.e. values in the few $\mathrm{GeV}$ range. Since as the total c.m. energy increases, $\mathrm{M}$ increases from 0.5 to $4 \mathrm{GeV}$, the region of $b>1 / M$ $(b<1 / M)$ corresponds to values of $b$ larger (smaller) than $2.5 \mathrm{GeV}^{-1}$, at low $\sqrt{s}$, down to $0.2 \mathrm{GeV}^{-1}$ for the highest $\sqrt{s}$ values. In other words, in the integration, small and large b-values are an energy dependent concept : at

very small $\sqrt{s}$, small b, i.e. $b<1 / M$ means values of $b$ less than $2.5 \mathrm{GeV}^{-1}$, 
whereas at very high energy large b-values mean $b>0.2 \mathrm{GeV}$. We shall now start studying $h(b ; M \Lambda)$ in the frozen $\alpha_{s}$ case, and distinguish three cases :

1. $b M<1$

2. $b M>1, b a \Lambda<1$

3. $b M>1, b a \Lambda>1$

In order to obtain a closed form expression to better study the function, we shall adopt the following approximations :

$$
\begin{aligned}
& \alpha_{s}(k<a \Lambda) \equiv \bar{\alpha}_{s}=\frac{12 \pi}{27 \ln \left(a^{2}\right)} \\
& \alpha_{s}(k>a \Lambda)=\frac{12 \pi}{27 \ln \frac{k^{2}}{\Lambda^{2}}} \\
& \ln \frac{M+\sqrt{M^{2}-k^{2}}}{M-\sqrt{M^{2}-k^{2}}} \approx 2 \ln \frac{2 M}{k} \quad k \approx 0 \text { and } \\
& \ln \frac{M+\sqrt{M^{2}-k^{2}}}{M-\sqrt{M^{2}-k^{2}}} \approx 2 \ln \frac{M}{k} \quad \text { for } k \text { values not in the infrared region. } \\
& 1-J_{0}(x)=\frac{x^{2}}{4} \quad x<1 \\
& 1-J_{0}(x)=1 \quad x>1
\end{aligned}
$$

Then, one can break the integral from $0 \rightarrow M$ into various intervals in which one can approximate the integrand and perform the integration. According to the three cases indicated above, one then obtains the following approximate expression :

$$
\begin{aligned}
b M & <1 \\
h(b, M, \Lambda) & =\frac{2 c_{F}}{\pi}\left[\bar{\alpha}_{s} \frac{b^{2}}{2} \int_{0}^{a \Lambda} k d k \ln \frac{2 M}{k}+\bar{b} \frac{b^{2}}{4} \int_{a \Lambda}^{M} k d k \frac{\ln \frac{M}{k}}{\ln \frac{k}{\Lambda}}\right] \\
& =\frac{2 c_{F}}{\pi}\left\{\bar{\alpha}_{s} \frac{b^{2} \Lambda^{2} a^{2}}{8}\left[1+2 \ln \frac{2 M}{a \Lambda}\right]+\right. \\
& \left.+\bar{b} \frac{b^{2} M^{2}}{8}\left\{\frac{a^{2} \Lambda^{2}}{M^{2}}-1+2 \frac{\Lambda^{2}}{M^{2}} \ln \frac{M}{\Lambda}\left[\operatorname{li}\left(\frac{M^{2}}{\Lambda^{2}}\right)-\operatorname{li}\left(a^{2}\right)\right]\right\}\right\}
\end{aligned}
$$


For $b M>1$, one distinguishes between two cases : $b$ larger or smaller than $1 / a \Lambda$, so that the integral can now be divided as follows :

$$
\begin{aligned}
\text { for } \quad \frac{1}{M} & <b<\frac{1}{a \Lambda} \\
h(b, M, \Lambda)= & \frac{2 c_{F}}{\pi}\left[\bar{\alpha}_{s} \frac{b^{2}}{2} \int_{0}^{a \Lambda} k d k \ln \frac{2 M}{k}+\bar{b} \frac{b^{2}}{4} \int_{a \Lambda}^{\frac{1}{b}} k d k \frac{\ln \frac{M}{k}}{\ln \frac{k}{\Lambda}}+\bar{b} \int_{\frac{1}{b}}^{M} \frac{d k}{k} \frac{\ln \frac{M}{k}}{\ln \frac{k}{\Lambda}}\right] \\
= & \frac{2 c_{F}}{\pi}\left[\bar{\alpha}_{s} \frac{b^{2} \Lambda^{2} a^{2}}{8}\left[1+2 \ln \frac{2 M}{a \Lambda}\right]+\right. \\
& \bar{b} \frac{b^{2} \Lambda^{2}}{8}\left\{a^{2}-\frac{1}{b^{2} \Lambda^{2}}+2 \ln \frac{M}{\Lambda}\left[l i\left(\frac{1}{b^{2} \Lambda^{2}}\right)-l i\left(a^{2}\right)\right]\right\}+ \\
& \left.\bar{b}\left[\ln \frac{M}{\Lambda} \ln \frac{\ln \frac{M}{\Lambda}}{\ln \frac{1}{b \Lambda}}-\ln (M b)\right]\right]
\end{aligned}
$$

or as

$$
\begin{aligned}
\text { for } \quad \frac{1}{M} & <\frac{1}{a \Lambda}<b \\
h(b, M, \Lambda)= & \frac{2 c_{F}}{\pi}\left[\bar{\alpha}_{s} \frac{b^{2}}{2} \int_{0}^{\frac{1}{b}} k d k \ln \frac{2 M}{k}+2 \bar{\alpha}_{s} \int_{\frac{1}{b}}^{a \Lambda} \frac{d k}{k} \ln \frac{M}{k}+\bar{b} \int_{a \Lambda}^{M} \frac{d k}{k} \frac{\ln \frac{M}{k}}{\ln \frac{k}{\Lambda}}\right] \\
= & \frac{2 c_{F}}{\pi}\left[\frac{\bar{\alpha}_{s}}{8}[1+2 \ln (2 M b)]+\right. \\
& 2 \bar{\alpha}_{s}\left\{\ln (M b) \ln (a \Lambda b)-\frac{1}{2} \ln ^{2}(a \Lambda b)\right\}+ \\
& \left.\bar{b}\left[\ln \frac{M}{\Lambda} \ln \frac{\ln \frac{M}{\Lambda}}{\ln a}-\ln \frac{M}{a \Lambda}\right]\right]
\end{aligned}
$$

The last decomposition is the one to use to study the large b limit, whereas the first one corresponds to the small $b$ limit.

For the singular $\alpha_{s}$ case we adopt similar approximations, except that now

$$
\begin{aligned}
& \alpha_{s}\left(k<N_{p} \Lambda\right)=\bar{b}\left(\frac{\Lambda}{k}\right)^{2 p} \\
& \alpha_{s}\left(k>N_{p} \Lambda\right)=\frac{\bar{b}}{\ln \left(\frac{k^{2}}{\Lambda^{2}}\right)}
\end{aligned}
$$


where $N_{p}=\left(\frac{1}{p}\right)^{1 / 2 p}$ is a number of order unity. For $\mathrm{p}=1 / 2$, indeed $N_{p}=2$ and the two regions, small and large $\mathrm{k}$, coincide with those in the frozen $\alpha_{s}$ case with $\mathrm{a}=2$. The expressions for $h(b ; M, \Lambda)$ in this case become :

$$
\begin{aligned}
b & <\frac{1}{M} \\
h(b ; M, \Lambda)= & \frac{2 c_{F}}{\pi}\left[\bar{b} \frac{b^{2}}{2}(\Lambda)^{2 p} \int_{0}^{\Lambda N_{p}} \frac{d k}{k^{2 p-1}} \ln \frac{2 M}{k}+\bar{b} \frac{b^{2}}{4} \int_{\Lambda N_{p}}^{M} k d k \frac{\ln \frac{M}{k}}{\ln \frac{k}{\Lambda}}\right] \\
= & \frac{2 c_{F}}{\pi}\left[\frac{\bar{b}\left(N_{p}^{2}\right)^{1-p}}{8(1-p)} b^{2} \Lambda^{2}\left(2 \ln \frac{2 M}{\Lambda N_{p}}+\frac{1}{1-p}\right)+\right. \\
& \left.\frac{\bar{b}}{8} b^{2} M^{2}\left\{\frac{N_{p}^{2} \Lambda^{2}}{M^{2}}-1+\frac{\Lambda^{2}}{M^{2}} 2 \ln \frac{M}{\Lambda}\left[\operatorname{li}\left(\frac{M^{2}}{\Lambda^{2}}\right)-\operatorname{li}\left(N_{p}^{2}\right)\right]\right\}\right]
\end{aligned}
$$

and for the $b M>1$ case one will have the two possibilities,

$$
\begin{aligned}
\text { for } \frac{1}{M}< & b<\frac{1}{N_{p} \Lambda} \\
h(b, M, \Lambda)= & \frac{2 c_{F}}{\pi}\left[\bar{b} \frac{b^{2}}{2} \Lambda^{2 p} \int_{0}^{N_{p} \Lambda} \frac{d k}{k^{2 p-1}} \ln \frac{2 M}{k}+\bar{b} \frac{b^{2}}{4} \int_{N_{p} \Lambda}^{\frac{1}{b}} k d k \frac{\ln \frac{M}{k}}{\ln \frac{k}{\Lambda}}+\bar{b} \int_{\frac{1}{b}}^{M} \frac{d k}{k} \frac{\ln \frac{M}{k}}{\ln \frac{k}{\Lambda}}\right] \\
= & \frac{2 c_{F}}{\pi}\left[\bar{b} \frac{b^{2} \Lambda^{2}}{8} \frac{\left(N_{p}^{2}\right)^{1-p}}{1-p}\left(2 \ln \frac{2 M}{N_{p} \Lambda}+\frac{1}{1-p}\right)+\right. \\
& \frac{\bar{b}}{8}\left\{N_{p}^{2} b^{2} \Lambda^{2}-1+2 \ln \frac{M}{\Lambda} b^{2} \Lambda^{2}\left[\operatorname{li}\left(\frac{1}{b^{2} \Lambda^{2}}\right)-\operatorname{li}\left(N_{p}^{2}\right)\right]\right\}+ \\
& \left.\bar{b}\left[\ln \frac{M}{\Lambda} \ln \frac{\ln \frac{M}{\Lambda}}{\ln \frac{1}{b \Lambda}}-\ln (M b)\right]\right]
\end{aligned}
$$

and the other case

$$
\begin{aligned}
b>\frac{1}{N_{p} \Lambda} & >\frac{1}{M} \\
h(b, M, \Lambda) & =\frac{2 c_{F}}{\pi}\left[\bar{b} \frac{b^{2} \Lambda^{2 p}}{2} \int_{0}^{\frac{1}{b}} \frac{d k}{k^{2 p-1}} \ln \frac{2 M}{k}+2 \bar{b} \Lambda^{2 p} \int_{\frac{1}{b}}^{N_{p} \Lambda} \frac{d k}{k^{2 p+1}} \ln \frac{M}{k}+\bar{b} \int_{N_{p} \Lambda}^{M} \frac{d k}{k} \frac{\ln \frac{M}{k}}{\ln \frac{k}{\Lambda}}\right] \\
& =\frac{2 c_{F}}{\pi}\left[\frac{\bar{b}}{8(1-p)}\left(b^{2} \Lambda^{2}\right)^{p}\left[2 \ln (2 M b)+\frac{1}{1-p}\right]+\right.
\end{aligned}
$$




$$
\begin{aligned}
& \frac{\bar{b}}{2 p}\left(b^{2} \Lambda^{2}\right)^{p}\left[2 \ln (M b)-\frac{1}{p}\right]+\frac{\bar{b}}{2 p N_{p}^{2 p}}\left[-2 \ln \frac{M}{\Lambda N_{p}}+\frac{1}{p}\right]+ \\
& \left.\bar{b} \ln \frac{M}{\Lambda}\left[\ln \frac{\ln \frac{M}{\Lambda}}{\ln N_{p}}-1+\frac{\ln N_{p}}{\ln \frac{M}{\Lambda}}\right]\right]
\end{aligned}
$$

This approximation is reasonably accurate, as one can see from Figs.12 13 , where we have plotted both the approximate and the exact expressions from the above equations for the two different models for $\alpha_{s}$. 


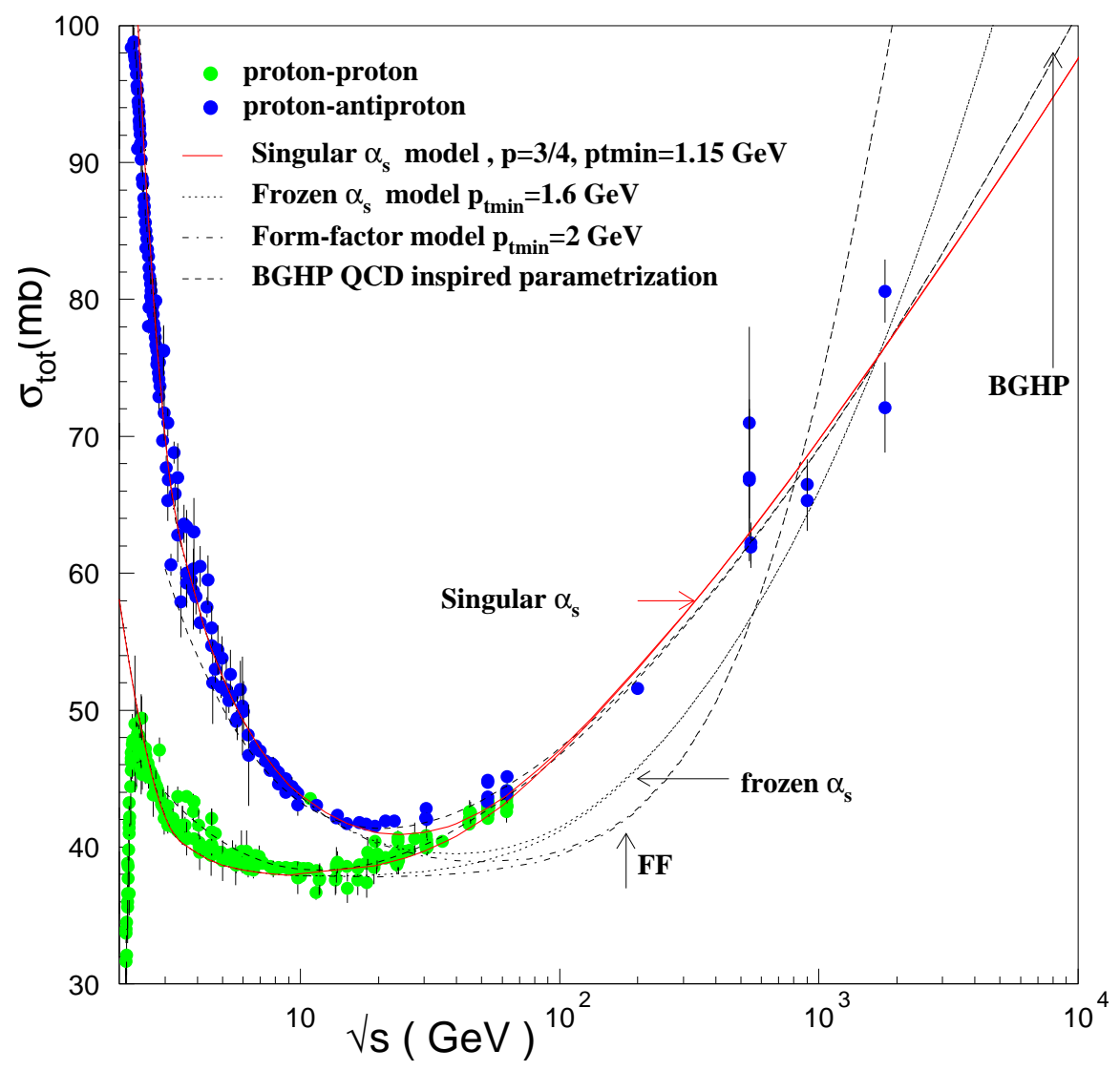

Figure 11: Total $p-p$ and $\bar{p} p$ cross-sections and comparison with various models 


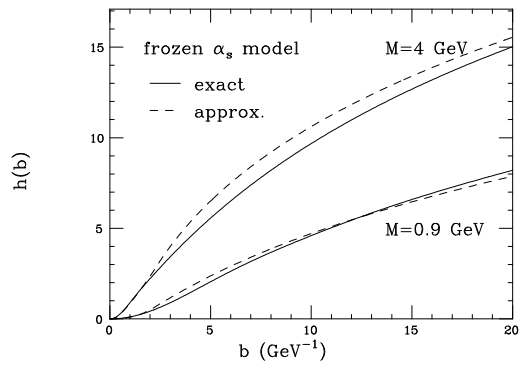

Figure 12: Comparison between the approximate and the actual numerical integration for $h(b, M, \Lambda)$ for various values for $\mathrm{M}$, in the frozen $\alpha_{s}$ model

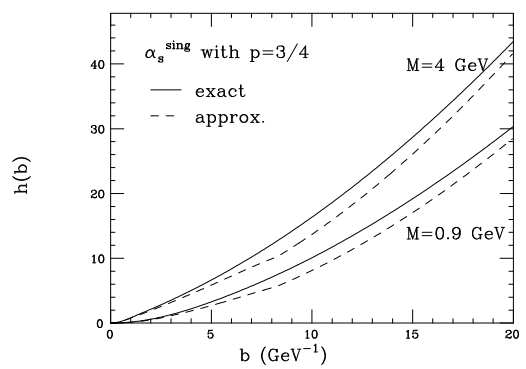

Figure 13: Comparison between the approximate and the actual numerically computed expression for $h(b, M, \Lambda)$ for the singular $\alpha_{s}$ model 


\section{References}

[1] Y.L. Dokshitzer, Perturbative QCD Theory (includes our knowledge of $\left.\alpha_{s}\right)$, Plenary Talk at ICHEP 1998, Vancouver, hep-ph/9812252.

[2] A. Corsetti, A. Grau, G. Pancheri and Y.N. Srivastava, Phys. Lett. B382 (1996) 282

[3] G. Pancheri-Srivastava and Y.N. Srivastava, Phys. Rev. D15 (1977) 2915.

[4] Yu.L. Dokshitzer, D.I. D’Yakonov and S.I. Troyan, Phys. Lett. B79 (1978) 269.

[5] G. Parisi and R. Petronzio, Nucl. Phys. B154 (1979) 427.

[6] P. Chiappetta and M. Greco, Nucl. Phys. B199 (1982) 77.

[7] D. Cline, F. Halzen and J. Luthe, Phys. Rev. Lett. 31 (1973) 491.

[8] T. Gaisser and F. Halzen, Phys. Rev. Lett. 54 (1985) 1754.

[9] G. Pancheri and Y. Srivastava, Phys. Lett. B158 (1986) 402.

[10] L. Durand and H. Pi, Phys. Rev. Lett. 58 (1987) 303.

[11] A. Capella, J. Kwiecinsky, J. Tran Thanh, Phys. Rev. Lett. 58 (1987) 2015.

[12] M.M. Block, F. Halzen, B. Margolis, Phys. Rev. D45 (1992) 839.

[13] M. Glück, E. Reya and A. Vogt, Phys. Rev. D46 (1992) 1973.

[14] F. Halzen, A.D. Martin and D.M. Scott, Phys. Rev. D25 (1982) 754; Phys. Lett. B112 (1982) 160.

[15] G. Altarelli, R.K. Ellis, M. Greco, and G. Martinelli, Nucl. Phys. B246 (1984) 12 .

[16] J.L. Richardson, Phys. Lett. B82 (1979) 272. 
[17] A. Nakamura, G. Pancheri and Y. Srivastava, Zeit. Phys. C21 (1984) 243.

[18] Baltrusaitis et al., Phys. Rev. Lett. 52 (1984) 1380.

M. Honda et al., Phys. Rev. Lett. 70 (1993) 525.

[19] R. Engel, T.K. Gaisser, P. Lipari and T. Stanev, Phys. Rev. D58 (1998) 014019 .

[20] Arnison et al., Phys. Lett. B128 (1983) 336.

R. Battiston et al., Phys. Lett. B117 (1982) 126.

Augier et al., Phys. Lett. B344 (1994) 451.

Bozzo et al., Phys. Lett. B147 (1984) 392.

Alner et al., Z. Phys. C32 (1986) 153.

[21] Amos et al., Phys. Rev. Lett. 68 (1991) 2433.

Abe et al., Phys. Rev. D50 (1993) 5550.

[22] M. Block, E. Gregores, F. Halzen and G. Pancheri, Phys. Rev. D58 (1998) 17503.

[23] A. Donnachie and P.V. Landshoff, Phys. Lett. B296 (1992) 227. 